\title{
Helium nanodroplet assisted synthesis of bimetallic Ag@Au nanoparticles with tunable localized surface plasmon resonance ${ }^{\star}$
}

\author{
Florian Lackner ${ }^{1, a}$, Alexander Schiffmann ${ }^{1}$, Maximillian Lasserus ${ }^{1}$, Roman Messner ${ }^{1}$, Martin Schnedlitz ${ }^{1}$, \\ Harald Fitzek ${ }^{2}$, Peter Pölt ${ }^{2}$, Daniel Knez ${ }^{2}$, Gerald Kothleitner ${ }^{2}$, and Wolfgang E. Ernst ${ }^{1}$ \\ 1 Institute of Experimental Physics, Graz University of Technology, Petersgasse 16, 8010 Graz, Austria \\ 2 Institute of Electron Microscopy and Nanoanalysis \& Graz Centre for Electron Microscopy, \\ Graz University of Technology, Steyrergasse 17, 8010 Graz, Austria
}

Received 20 December 2018 / Received in final form 22 March 2019

Published online 21 May 2019

(C) The Author(s) 2019. This article is published with open access at Springerlink.com

\begin{abstract}
Nanoparticles with tunable localized surface plasmon resonance have been prepared by synthesis in helium nanodroplets. Subsequent surface deposition allows for the formation of substrates which can be employed for surface-enhanced Raman spectroscopy (SERS). The assembly of Ag@Au core@shell clusters of about $5 \mathrm{~nm}$ diameter in helium droplets with different $\mathrm{Ag}$ : Au ratio allows to tune the surface plasmon resonance between the plain $\mathrm{Ag}$ resonance at $447 \mathrm{~nm}$ and the Au resonance at $555 \mathrm{~nm}$. For the fabricated substrates only a single plasmon resonance is observed in the UV/vis absorption spectra. The prepared nanostructures have been functionalized with 4-methylbenzenethiol (4-MBT) molecules and SERS spectra have been recorded. The results demonstrate the potential of the helium droplet synthesis approach, opening up a new route to the formation of tailored plasmonic nanoparticles and functional nanostructures.
\end{abstract}

\section{Introduction}

Nano-sized particles have paved the way for exciting new research and applications in science and technology. Today, nanoparticles are ubiquitous and have found application in catalysis [1] and photovoltaics [2] as well as in chemistry [3], biology [4] and medicine [5]. Hybrid nanoparticles, in particular, play an important role in emerging applications as they allow to tailor particle characteristics by deliberately combining materials with desired magnetic, optical or chemical properties [6]. The great potential of such nanoparticles and nanostructures assembled thereof is calling for novel synthesis methods capable to rapidly explore a large variety of different material combinations. Here, we introduce the helium nanodroplet synthesis method as a novel approach for the production of functional hybrid nanoparticles with tailored plasmonic properties.

The advantages that come with the helium droplet approach are the (i) intrinsic inertness of the synthesis environment (liquid helium) that does not involve any solvents, surfactants or other chemicals, (ii) the large variety of dopants that can be combined and (iii) the cold environment $(0.37 \mathrm{~K})[7,8]$ that enables the production of nanoparticles with a core@shell configuration indepen-

* Contribution to the Topical Issue "Dynamics of Systems on the Nanoscale (2018)", edited by Ilko Bald, Ilia A. Solov'yov, Nigel J. Mason and Andrey V. Solov'yov.

a e-mail: florian.lackner@tugraz.at dent of the thermodynamically favored structure [9]. In the present study, we chose the $\mathrm{Ag} @ \mathrm{Au}$ system in order to demonstrate the controlled formation of plasmonic nanoparticles and nanostructures by helium droplet synthesis. However, in general the approach allows to select nanoparticle constituents from a large variety of materials.

Many of the outstanding optical properties of plasmonic materials are related to surface plasmon resonances or, in the case of coinage metal nanoparticles smaller than the wavelengths of visible light, localized surface plasmon resonances (LSPR). These plasmons can be understood as collective oscillations of electrons within the nanoparticle. The spectral position of a LSPR thereby depends strongly on the structure, shape and size of the particle [6]. The resonant interaction between an electromagnetic field and the surface plasmon causes a concentration of electromagnetic radiation close by the nanoparticle surface, an effect which forms the foundation of surface-enhanced Raman scattering (SERS): when a Raman active molecule is attached to the surface of a metal nanoparticle and exposed to electromagnetic radiation resonant with the LSPR it experiences a strongly enhanced field, which can boost the Raman signal by orders of magnitude [4].

Typically, $\mathrm{Au}$ or $\mathrm{Ag}$ nanostructures are used as SERS substrates, however, hybrid nanoparticles pose very promising building blocks for the preparation of novel SERS substrates for emerging and future applications $[6,10]$. A well characterized material combination in this context is represented by $\mathrm{Ag}-\mathrm{Au}$ hybrid nanoparticles [11], which we chose to demonstrate the capabilities of our 
synthesis approach. Previous studies on core@shell nanoparticles, with diameters larger than $10 \mathrm{~nm}$, have shown that for a Ag@Au as well as a Au@Ag configuration typically two separate plasmon resonances are observed, one close to the original Ag LSPR and the other close to the $\mathrm{Au}$ LSPR peak, their respective intensities depending on the Ag:Au ratio [12-17]. In the alloyed form, however, nanoparticles exhibit only a single plasmon resonance and its position depends on the $\mathrm{Ag}$ : $\mathrm{Au}$ ratio $[13,15,17,18]$. It has been shown that both structures are suitable for the preparation of SERS substrates [11,14,19].

With the helium droplet synthesis approach nanoparticles are formed when the droplets pass a pickup region, typically a cell in which a resistively heated oven establishes an increased vapor pressure of the desired dopant material. The subsequent arrangement of two of such pickup cells allows for the doping with two different materials. Under such conditions core@shell nanoparticles can be formed, where the material introduced first forms the core and the second the shell [20,21]. Subsequently, the particles can be deposited on any desired substrate, thereby the surrounding helium acts as a cushion that is stripped off upon impact, dissipating the released energy and resulting in a very soft deposition $[22,23]$.

In the recent years helium droplets have evolved from a pure spectroscopic matrix $[7,8]$ to a synthesis method for nanoparticles [24-28]. However, the vast majority of works dealing with nanoparticle synthesis is focused on the investigation of particle size, structure and morphology. Here, we expand the helium droplet synthesis approach into the field of plasmonics by presenting a new tool for the production of bimetallic plasmonic nanoparticles and nanostructures.

\section{Experimental}

\subsection{Nanoparticle synthesis}

The employed helium droplet apparatus has been described in reference [29]. Bimetallic nanoparticles are formed by guiding a helium droplet beam through two independent pickup zones at which enclosed, heated crucibles establish an increased vapor pressure of the desired dopant material. The employed helium droplets have a mean radius of about $40 \mathrm{~nm}$, obtained by using a nozzle temperature of $8 \mathrm{~K}$, a stagnation pressure of 20 bar and a $5 \mu \mathrm{m}$ diameter nozzle [30]. Upon passing the first pickup zone, loaded with Ag, the dopant atoms agglomerate inside the helium droplets. When passing the second pickup zone, loaded with Au, the agglomerated Ag clusters are covered by a $\mathrm{Au}$ shell [31]. Pure Ag and Au clusters are formed by heating only one crucible. After formation the nanoparticles are deposited on the desired substrates under UHV conditions in a subsequent chamber. Several substrates can be interchanged inside the apparatus without breaking the vacuum.

When metal atoms agglomerate, binding energy is released into the droplet, which is dissipated by the evaporation of He atoms. This process goes along with a decrease of the ${ }^{4} \mathrm{He}$ yield in the quadrupole mass spectrometer at the end of the machine [30], which, thus, provides a convenient way to monitor the doping level. However, when applying this method to multiple dopants added subsequently to the droplets, differences in the binding energies have to be accounted for, which can be estimated by the bulk cohesive energies. In the case of $\mathrm{Ag}$ and $\mathrm{Au}$ clusters these energies correspond to $2.95 \mathrm{eV}$ and $3.81 \mathrm{eV}$, respectively [32]. Thus, the depletion caused by Ag clusters formed in helium droplets is about $23 \%$ lower than for $\mathrm{Au}$ clusters. Consequently, the three selected compositions obtained by setting the attenuation of the droplet beam flux to $30 \% \mathrm{Ag} / 20 \% \mathrm{Au}, 20 \% \mathrm{Ag} / 30 \% \mathrm{Au}$ and $15 \% \mathrm{Ag} / 35 \% \mathrm{Au}$ correspond to a corrected $\mathrm{Ag}: \mathrm{Au}$ composition ratio in terms of number of atoms of approximately $1.94,0.86$, and 0.55 , respectively. However, for the sake of convenience we refer to these compositions as $2: 1$, 1:1 and 1:2 in the following. The estimated average total number of atoms (sum of $\mathrm{Ag}$ and $\mathrm{Au}$ atoms) introduced into the helium droplet remains fairly constant for different Ag:Au ratios and corresponds to about 2800 atoms per nanoparticle.

\section{$2.2 \mathrm{UV} /$ vis absorption spectroscopy}

$\mathrm{UV} /$ vis absorption spectra were recorded employing a Shimadzu UV-1800 spectrophotometer. The nanoparticles were deposited by introducing a fused silica microscope slide (Corning 7980, fused silica, $1 \mathrm{~mm}$ thickness) into the beam. The slide is subsequently taken out of the vacuum and placed into the spectrophotometer to acquire the spectra shown in Figure 4, using a second fused silica slide as reference.

\subsection{Transmission electron microscopy (TEM)}

For TEM experiments the clusters are deposited on TEM grids (Ted Pella, Inc., Prod. No. 01824), covered with an amorphous carbon film with a thickness of $3 \mathrm{~nm}$. The TEM images shown in Figure 1 are recorded with a $120 \mathrm{kV}$ LaB6 FEI Tecnai 12 transmission electron microscope, equipped with a Bio Scan Camera (Gatan, Model 792). High-resolution scanning transmission electron microscopy (HR-STEM) is performed using a probecorrected FEI Titan3 G2 60-300 instrument. The STEM images shown in Figure 3 have been acquired using a highangular annular dark-field (HAADF) imaging detector. Note that during transport from the helium droplet apparatus to the TEM instrument the samples are exposed to air for a short period of time, which should have no effect on the deposited $\mathrm{Ag}$ and $\mathrm{Au}$ materials.

\subsection{Raman spectroscopy}

After recording the absorption spectra of nanoparticles deposited on the fused silica glass slides, the substrates were functionalized with 4-methylbenzenethiol (4-MBT) molecules. The functionalization is done by immersing the 


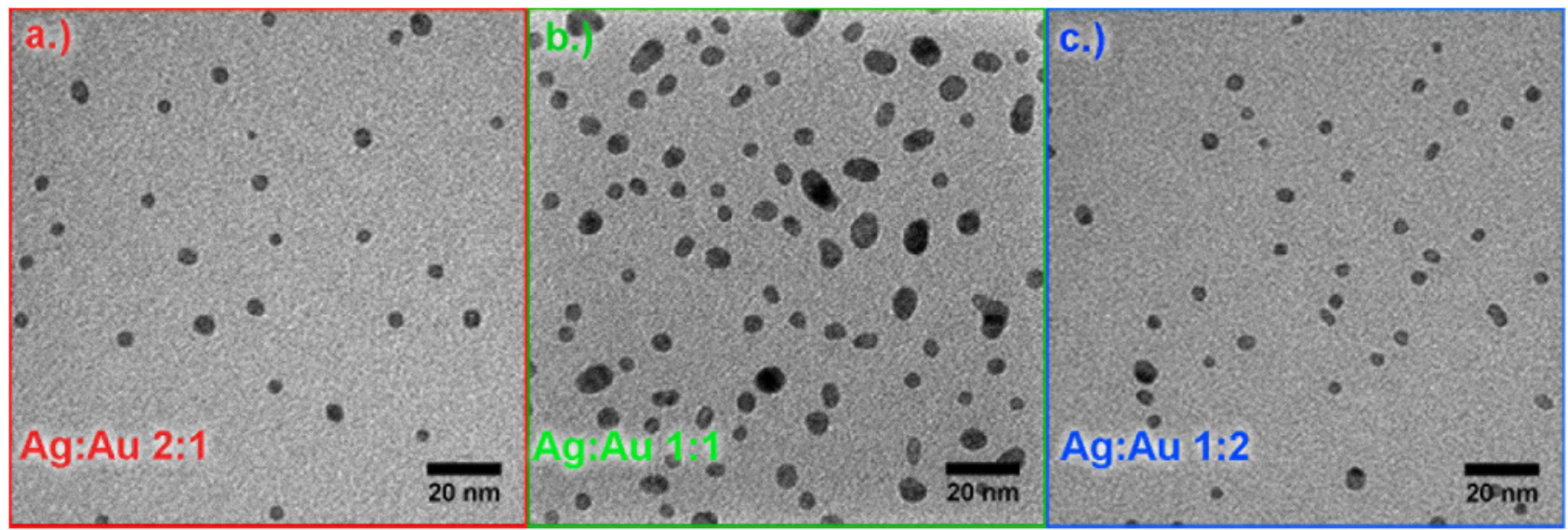

Fig. 1. Bright-field (BF) transmission electron microscopy (TEM) images of the produced nanoparticles with Ag:Au ratios of 2:1 (red frame, a), 1:1 (green frame, b) and 1:2 (blue frame, c). Particles have been deposited for 30 min (2:1), 4h (1:1) and $30 \mathrm{~min}(1: 2)$. The images show an overview of the deposited particles (scale bar $20 \mathrm{~nm}$ ). Coagulation of a fraction of the particles is observed for long deposition times (middle image).

substrate in a $0.1 \mathrm{mM}$ solution of 4 -MBT in DI water for $1 \mathrm{~h}$. Excess 4 -MBT is removed by subsequent immersion in ethanol. Raman spectra were acquired employing a LabRam HR 800 spectrometer. The used laser wavelengths (and powers) are $473 \mathrm{~nm}(12.5 \mathrm{~mW}), 532 \mathrm{~nm}$ $(12.5 \mathrm{~mW})$ and $633 \mathrm{~nm}(15 \mathrm{~mW})$. A x10 Olympus MplanN (N.A. 0.25) objective was used to focus the laser onto the sample. For every substrate 64 spectra were measured across an area of $500 \times 500 \mu \mathrm{m}$ with an acquisition time of $8 \mathrm{~s}$ (and 2 accumulations) per spectra. The shown Raman spectra in Figures 5 and 6 correspond to the average of these 64 spectra. Enhancement factors EF were determined according to the definition and procedure provided by Le Ru et al. [33]:

$$
E F=\frac{I_{\text {SERS }} N_{\text {Ref }}}{I_{\text {Ref }} N_{\text {SERS }}}=\frac{I_{\text {SERS }} c_{\text {Ref }} H_{\text {eff }}}{I_{\text {Ref }} A \mu_{S}} .
$$

All band intensities $\left(I_{\mathrm{SERS}}, I_{\mathrm{Ref}}\right)$ were determined using the peak fit routine of the LabSpec 6 software. Reference measurements were done for two different solutions (ethanol and isopropanol; molar concentration $c_{\text {Ref }}=0.5 \mathrm{M}$ ) in order to obtain reference intensities for all bands without interference from the solvent. The effective height of the scattering volume $H_{\mathrm{eff}}=174 \mu \mathrm{m}$ was determined as described in the supplementary information of Le $\mathrm{Ru}$ et al. [33] The surface density $\mu_{S}$ of 4 -MBT is taken from Camargo et al. [34] $\left(\mu_{S}=5.3 \mathrm{~nm}^{-2}\right)$. From the TEM images, which show a projection of the deposited nanoparticles, the ratio of the surface area of the metallic nanoparticles to the total substrate area is estimated as $A=0.58$ by assuming spherical particles.

\section{Results and discussion}

Figure 1 shows a survey of bright-field (BF) images of the prepared bimetallic Ag@Au core@shell particles with Ag:Au ratios adjusted to 2:1 (red frame, a), 1:1 (green frame, b) and 1:2 (blue frame, c), recorded by transmission
Table 1. Summary of the determined surface coverage (sc) per hour and the mean particle diameter $d$ estimated by assuming spherical particles for nanoparticles with Ag:Au ratios of 2:1, 1:1 and 1:2, obtained from the recorded TEM images. In addition, the full width at half maximum (FWHM) of the particle size distribution for each $\mathrm{Ag}: \mathrm{Au}$ ratio, shown in Figure 2, is listed.

\begin{tabular}{llll}
\hline Ag:Au ratio & sc per hour & $d(\mathrm{~nm})$ & FWHM \\
\hline $2: 1$ & $(4.7 \pm 0.6) \%$ & 4.7 & 2.0 \\
$1: 1$ & $(3.6 \pm 0.2) \%$ & 6.5 & 4.9 \\
$1: 2$ & $(4.7 \pm 0.4) \%$ & 4.4 & 2.2 \\
\hline
\end{tabular}

electron microscopy (TEM). Note that the ratio refers to the estimated ratio of the number of atoms, which can be easily controlled by adjusting the temperatures of the pickup-ovens [31].

The nanoparticles were deposited on standard amorphous carbon TEM grids immediately after deposition on fused silica substrates at the same helium droplet source conditions in order to reveal insight into the surface coverage, particle size and structure. The intensity in the shown $\mathrm{BF}$ images depends on the atomic number $(\mathrm{Z})$, i.e. bright areas correspond to $\mathrm{Ag}$ rich zones whereas dark regions indicate $\mathrm{Au}$ rich zones. Note that only image (b) has been obtained upon deposition for four hours, similar to the samples used in the optical experiments. Nanoparticles in images (a) and (c) have been deposited for $30 \mathrm{~min}$ on the TEM grids. The comparison of particles prepared with different deposition times reveals insight into coagulation processes that can proceed upon deposition: while for low surface coverage nearly all particles remain separated, upon $4 \mathrm{~h}$ of continuous deposition the surface coverage is high enough that a fraction of the particles coagulates and forms larger structures.

The obtained surface coverages (sc) and mean particle diameters $d$ (estimated by assuming spherical particles) are listed in Table 1. Note that in order to ease 


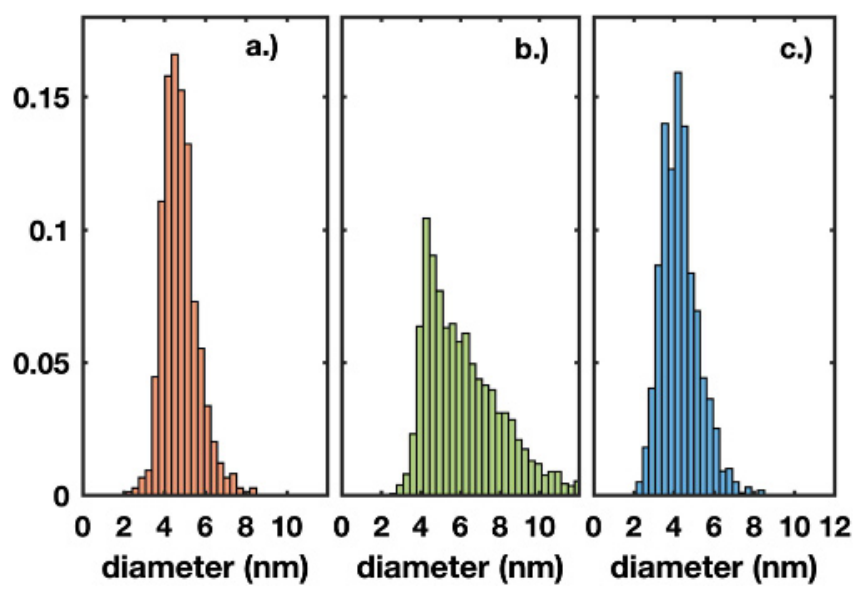

Fig. 2. Size distributions for particles with an Ag:Au ratio of 2:1 (a), 1:1 (b) and 1:2 (c). Note that, while panels (a) and (c) show a distribution of particles deposited for $30 \mathrm{~min}$, panel (b) shows the distribution of particles deposited for $4 \mathrm{~h}$, similar to the TEM images in Figure 1.

a comparison, the surface coverages have been scaled to one hour of deposition, neglecting the statistic nature of the process, i.e. particles may be deposited on top of each other. It can be seen that coagulation, which becomes relevant in case of the longer deposition time for the 1:1 ratio particles, manifests in an increase of the estimated mean particle diameter and, to some extent, also in a decrease of the surface coverage.

The particle size distributions are shown in Figure 2, the corresponding full width at half maximum (FWHM) of each distribution is listed in Table 1. Here, the effect of particle coagulation becomes obvious by comparing the distributions in panels (a) and (c), where particles have been deposited for 30 min, to the distribution in panel (b) for which the particles have been deposited for $4 \mathrm{~h}$. Coagulation leads to a significant broadening of the distribution towards larger particle sizes. We note that the coagulation process may differ between the amorphous carbon TEM grid substrate and the fused silica glass slides. However, it has to be considered in the interpretation of the $\mathrm{UV} /$ vis absorption spectra that a fraction of larger particles, which may not retain a core@shell structure, can contribute to the signal.

While the images in Figure 1 allow a characterization of the particle sizes and surface coverages, the resolution is too low in order to reveal insight into structural details, in particular, the core@shell structure of the particles. In order to provide further insight into the structure of nanoparticles in the $5 \mathrm{~nm}$ size regime formed by helium droplet synthesis we present selected highresolution images in Figure 3 . The shown particles have been produced in a previous series of experiments under similar helium droplet source conditions, however, the size of the shown particles and, in particular, the Ag:Au composition is the same as in the present study. Further details about the structure of nanoparticles comprising a $\mathrm{Ag}: \mathrm{Au}$ ratio of 1:1 can be found in reference [20] (and the Supplementary Information of Ref. [20]). The shown

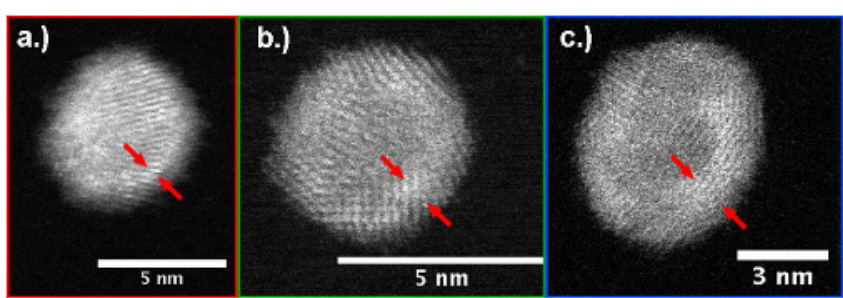

Fig. 3. High-angle annular dark-field (HAADF) images of representative core@shell nanoparticles from a separate series of experiments, recorded by a high-resolution scanning transmission electron microscope (HR-STEM). The Ag:Au ratios correspond to 2:1 (red frame, a), 1:1 (green frame, b) and 1:2 (blue frame, c). The Au shell layer is marked by red arrows.

images in Figure 3 correspond to high-angle annular darkfield (HAADF) images recorded by a high-resolution scanning transmission electron microscope (HR-STEM). The $\mathrm{Ag}: \mathrm{Au}$ ratios of the prepared nanoparticles correspond to 2:1 (red frame, a), 1:1 (green frame, b) and 1:2 (blue frame, c). The HAADF image intensity is proportional to the square of the atomic number $\left(\mathrm{Z}^{2}\right)$ [35]. Consequently, the image contrast within an individual nanoparticle represents zones that are rich of $\mathrm{Ag}$ (dark) or $\mathrm{Au}$ (bright).

It can be seen from the images in Figure 3 that even for the lowest $\mathrm{Au}$ doping level (Ag: $\mathrm{Au}$ of 2:1) a faint bright $\mathrm{Au}$ layer is visible at the surface (marked by red arrows). Consequently, it is concluded that even in the sub $5 \mathrm{~nm}$ size regime the helium droplet synthesis allows for the deposition of nanoparticles with a core@shell structure with extremely thin shell layers.

The optical properties of the prepared nanoparticles, deposited for $4 \mathrm{~h}$ each onto fused silica substrates, have been characterized employing UV/vis absorption spectroscopy. The recorded spectra are shown in Figure 4. Plain Ag (blue) and Au (red) nanoparticles serve as a reference, both exhibiting the characteristic LSPR of small, spherical nanoparticles on fused silica $[11,36]$ peaking at $447 \mathrm{~nm}$ for $\mathrm{Ag}$ and $555 \mathrm{~nm}$ for Au. The figure also features spectra of deposited core@shell nanoparticles with $\mathrm{Ag}: \mathrm{Au}$ ratios adjusted to 1:2 (orange), 1:1 (black) and 2:1 (green). Only a single LSPR peak is observed, which becomes increasingly red-shifted with increasing Au content, accompanied by decreasing intensity. For high Ag concentration (Ag:Au of 2:1) the LSPR feature resembles the $\mathrm{Ag}$ resonance, red-shifted to $484 \mathrm{~nm}$. For medium (1:1) and high $\mathrm{Au}$ content (1:2) the red-shift is stronger and the LSPR peak maxima are found at $499 \mathrm{~nm}$ and $518 \mathrm{~nm}$, respectively.

Previous studies on bimetallic $\mathrm{Ag}-\mathrm{Au}$ nanoparticles relied on different preparation processes such as coating of the core metal using radiation chemistry [13], laser ablation [16], or solution phase synthesis $[15,17,18]$. Measured absorption spectra indicated that core@shell nanoparticles exhibit two plasmon resonances, one close to the resonance of the pure $\mathrm{Au}$ particle and one close to the resonance of the pure Ag particle. It required the transformation into an alloy state to obtain continuous shifts of the then single resonance between the pure $\mathrm{Au}$ and $\mathrm{Ag}$ absorption wavelength, the shift depending on the $\mathrm{Au} / \mathrm{Ag}$ molar ratios. 


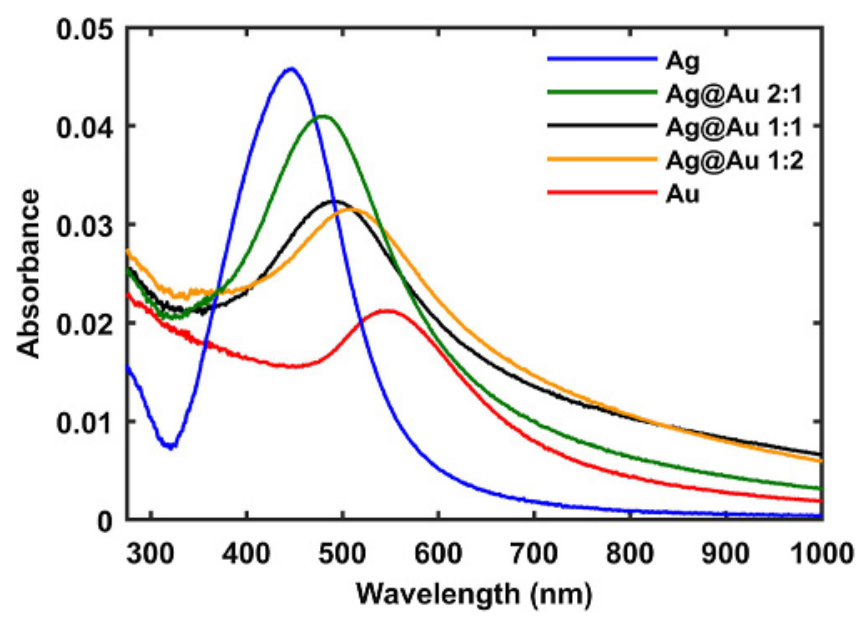

Fig. 4. UV/vis absorption spectra of the prepared $\mathrm{Ag}, \mathrm{Au}$ and Ag@Au core@shell nanoparticles deposited for $4 \mathrm{~h}$ on fused silica substrates. The Ag: Au ratios have been adjusted to 1:2 (orange), 1:1 (black) and 2:1 (green). Spectra of bare Ag and Au particles are shown in blue and red, respectively. The LSPR peak maximum shifts from $447 \mathrm{~nm}(\mathrm{Ag})$ to $555 \mathrm{~nm}(\mathrm{Au})$ with increasing $\mathrm{Au}$ content, accompanied by an intensity decrease.

The size of the nanoparticles in these previous studies was of $15 \mathrm{~nm}$ diameter or larger $[12,13,15,16]$. As the different absorption behavior for bimetallic core@shell versus alloy nanoparticles was even used as diagnostic tool to distinguish the two states, Kuzma et al. [37] discuss optical properties of binary compound plasmonic nanoparticles (see also Ref. [38]). They found that optical absorption spectra alone are not sufficient to predict structural arrangements.

In the case of helium droplet synthesis it is known that the nanoparticles that are formed in the droplet and subsequently deposited are of core@shell structure [9,20,31], which becomes also evident from Figure 3. However, as suggested by the particle size distribution in Figure 2, the optical spectrum does not only originate from the initially deposited core@shell particles but may also contain a contribution from larger, coagulated particles. The sum of both contributions leads to the relatively broad single plasmon resonance feature that is observed in the absorption spectrum. Furthermore, at a surface coverage of about $15 \%$ the coupling of LSPR modes between individual particles becomes important [39], the corresponding features may contribute to the spectrum in the red part of the spectra in Figure 4 at longer wavelength [40].

Here, single particle plasmon spectroscopy based on electron energy loss spectroscopy (EELS) [41,42] could reveal more insight into the plasmonic properties of individual particles as UV/vis absorption spectroscopy can only probe the average spectrum of a large number of particles. In particular, helium droplet synthesis would allow for the study of particles with very thin shell layers, such as shown in Figure 3a.

The prepared particles have a size of only $\sim 5 \mathrm{~nm}$, corresponding to about 2800 atoms on average, and, thus, the increased surface to volume ratio becomes important. Considering a Mackay icosahedron structure [43], a cluster with 10 closed shells consisting of 2869 has 812 surface atoms, which corresponds to a surface to volume ratio of about 1:2.5. Thus, in this idealized scenario a $\mathrm{Ag} @ \mathrm{Au}$ core@shell particle consisting of 2869 atoms in total with a Ag:Au ratio of $2.5: 1$ could be surrounded only by one atomic monolayer of $\mathrm{Au}$. From this estimation it becomes evident that at lowest $\mathrm{Au}$ doping levels the shell layer is extremely thin, in agreement with the nanoparticle shown in Figure 3a. This represents an interesting situation, where common theoretical models for the calculation of plasmon resonances that predict two disjunct peaks in the spectrum for core@shell nanoparticles [37,44] may cease to be valid as properties such as surface roughness, surface diffusion and atomic interdiffusion processes become important [45]. Furthermore, at around $5 \mathrm{~nm}$ various different cluster structures and morphologies become possible [25], which deviate from the idealized models.

Having characterized the nanoparticles and their optical properties, we can now proceed to analyze their capabilities as substrates for surface-enhanced Raman spectroscopy (SERS). Therefore, the nanoparticles deposited on fused silica substrates were functionalized with 4-methylbenzenethiol (4-MBT). The Raman spectra obtained for plain $\mathrm{Ag}$ and $\mathrm{Au}$ nanoparticle substrates as well as for bimetallic particles are shown in Figure 5 for three different Raman laser wavelengths. Note that the $\mathrm{Ag}$ spectra for $532 \mathrm{~nm}$ and $633 \mathrm{~nm}$ in Figure 5 have been scaled by x0.2 and the $532 \mathrm{~nm} \mathrm{Au} \mathrm{an} \mathrm{Ag@Au} \mathrm{1:2} \mathrm{as} \mathrm{well} \mathrm{as}$ the $473 \mathrm{~nm} \mathrm{Ag@Au} \mathrm{2:1} \mathrm{spectra} \mathrm{have} \mathrm{been} \mathrm{scaled} \mathrm{by} \mathrm{x2.5.}$

From near-edge X-ray absorption fine structure (NEXAFS) spectroscopy it is known that the sulfur atom, situated at one end of the molecule, binds to the surface (in the ideal case of a planar $\mathrm{Au}(111)$ surface the benzene ring is tilted by $33^{\circ}$ with respect to the surface) $[46,47]$. Furthermore, 4 -MBT is known to form selfassembled monolayers on both $\mathrm{Ag}$ and $\mathrm{Au}$ surfaces [48].

The Raman spectrum in Figure 5 can be readily assigned based on comparison to literature $[34,49]$. The most intense peak at $1080 \mathrm{~cm}^{-1}$ corresponds to a combination of a phenyl breathing mode, a $\mathrm{CH}$ in-plane bending and CS stretching (7a vibrational mode). The intense peak at $1595 \mathrm{~cm}^{-1}$ corresponds to a phenyl ring stretching mode (8a vibrational mode). The three peaks in between these two intense features at $1182 \mathrm{~cm}^{-1}, 1378 \mathrm{~cm}^{-1}$, and $1486 \mathrm{~cm}^{-1}$ as well as the peak at $1698 \mathrm{~cm}^{-1}$ are assigned to in-plane phenyl ring vibrations. The broad structures observed in the left panel $(473 \mathrm{~nm})$ of Figure 5 for the three top most spectra and for the top spectrum in the middle $(532 \mathrm{~nm})$ panel correspond to residuals from the fused silica substrate, only the sharp lines are associated with signals from molecules.

Enhancement factors have been determined for the strongest Raman mode at $1080 \mathrm{~cm}^{-1}$ and are listed in Table 2. Note that the Raman enhancement factors have been determined by averaging over a $500 \mu \mathrm{m} \times 500 \mu \mathrm{m}$ area. Considering that the particles are close enough to coagulate, hot-spots between nearby nanoparticles are, presumably, present on the substrate, boosting the observed enhancement factors. It can be seen that the Ag nanoparticle substrate, which also exhibits the most intense LSPR, shows the highest enhancement. In general, 


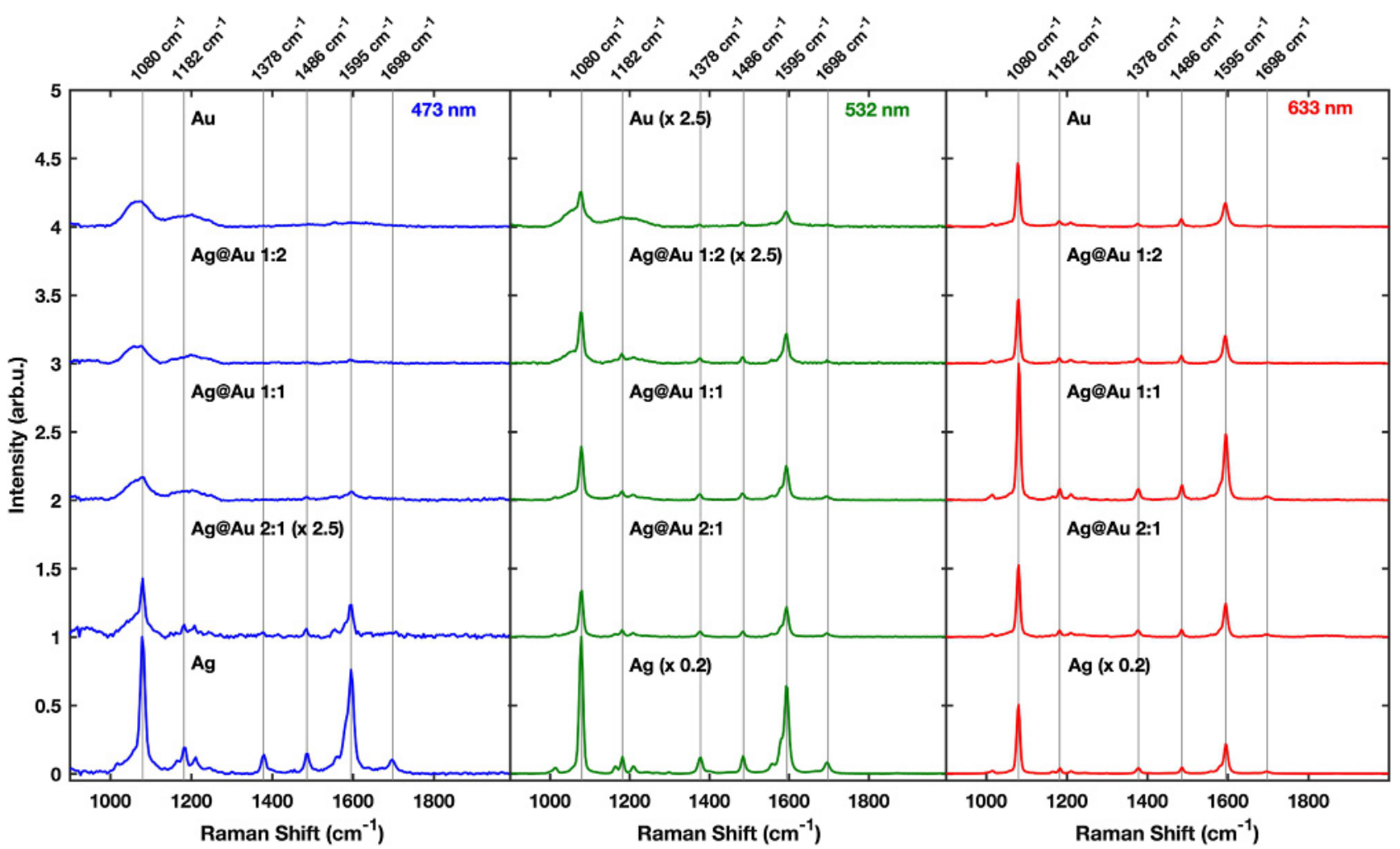

Fig. 5. Raman spectra recorded for the different nanoparticles functionalized with 4-methylbenzenethiol (4-MBT) molecules obtained by employing three different Raman laser wavelengths of $473 \mathrm{~nm}$ (blue), $532 \mathrm{~nm}$ (green) and $633 \mathrm{~nm}$ (red). The Raman spectrum obtained from 4-MBT attached to core@shell nanoparticle substrates with Ag:Au ratios of 2:1, 1:1 and 1:2 are shown together with spectra obtained from substrates consisting of plain $\mathrm{Au}$ and $\mathrm{Ag}$ particles.

Table 2. Enhancement factors determined for the Raman mode at $1080 \mathrm{~cm}^{-1}$ for nanoparticle substrates comprising $\mathrm{Ag}: \mathrm{Au}$ ratios of $2: 1,1: 1$ and $1: 2$ as well as for the plain $\mathrm{Ag}$ and $\mathrm{Au}$ nanoparticle substrates.

\begin{tabular}{lccl}
\hline $\mathrm{Ag}: \mathrm{Au}$ & $473 \mathrm{~nm}$ & $532 \mathrm{~nm}$ & $633 \mathrm{~nm}$ \\
\hline $1: 0$ & 1800 & 24000 & 30000 \\
$2: 1$ & 200 & 2100 & 6300 \\
$1: 1$ & 70 & 1700 & 12000 \\
$1: 2$ & Bld $^{*}$ & 800 & 5800 \\
$0: 1$ & Bld $^{*}$ & 300 & 5500 \\
\hline
\end{tabular}

${ }^{*}$ Bld: below limit of detection.

the enhancement factors are lowest for the blue laser $(473 \mathrm{~nm})$ and highest for the red laser $(633 \mathrm{~nm})$. The enhancement factors for the bimetallic substrates are situated in between the pure Ag and Au nanoparticle substrates, where the Ag@Au nanoparticles comprising a 1:1 $\mathrm{Ag}: \mathrm{Au}$ ratio show the next highest enhancement after the pure Ag nanoparticles. Note that the position of the LSPR maximum (which may be affected by the functionalization with 4-MBT [50]) does not necessarily have to coincide with the wavelength for maximum Raman enhancement [51], in particular, for substrates where individual nanoparticles are not completely isolated from each other.
An interesting aspect of the Raman spectra is the dependence of the Raman bands at $1595 \mathrm{~cm}^{-1}$ and $1698 \mathrm{~cm}^{-1}$ on the nanoparticle substrate, which differ greatly between the case where the molecules are attached to a $\mathrm{Au}$ or a $\mathrm{Ag}$ surface. For $4-\mathrm{MBT}$ on $\mathrm{Ag}$ it is known that the peak at $1595 \mathrm{~cm}^{-1}$ shows a characteristic asymmetry [34]. The origin of this effect is still under debate but it may either be caused by a broadening of the $8 \mathrm{a}$ mode or a selective enhancement of the $8 \mathrm{~b}$ mode, located about $15 \mathrm{~cm}^{-1}$ below. However, on $\mathrm{Au}$ substrates this effect is absent [49]. Furthermore, the $1698 \mathrm{~cm}^{-1}$ mode is only significantly enhanced for the Ag substrate [49]. Figure 6 shows a zoom into the corresponding region. It can be seen that the spectra gradually change their character from plain Ag to plain Au character with increasing $\mathrm{Au}$ shell thickness. Only when multiple $\mathrm{Au}$ shell layers are present (Ag:Au of 1:2, orange spectrum) the spectrum resembles the one obtained for molecules attached to plain $\mathrm{Au}$ nanoparticles. This indicates that even if coagulated, larger particles are formed on the glass substrate and contribute to the signal, in the case of nanoparticles with a $\mathrm{Ag}: \mathrm{Au}$ ratio of 1:2 they are still covered by a fully closed $\mathrm{Au}$ shell.

\section{Conclusions}

The present work demonstrates the capabilities of the helium droplet synthesis approach for the production 


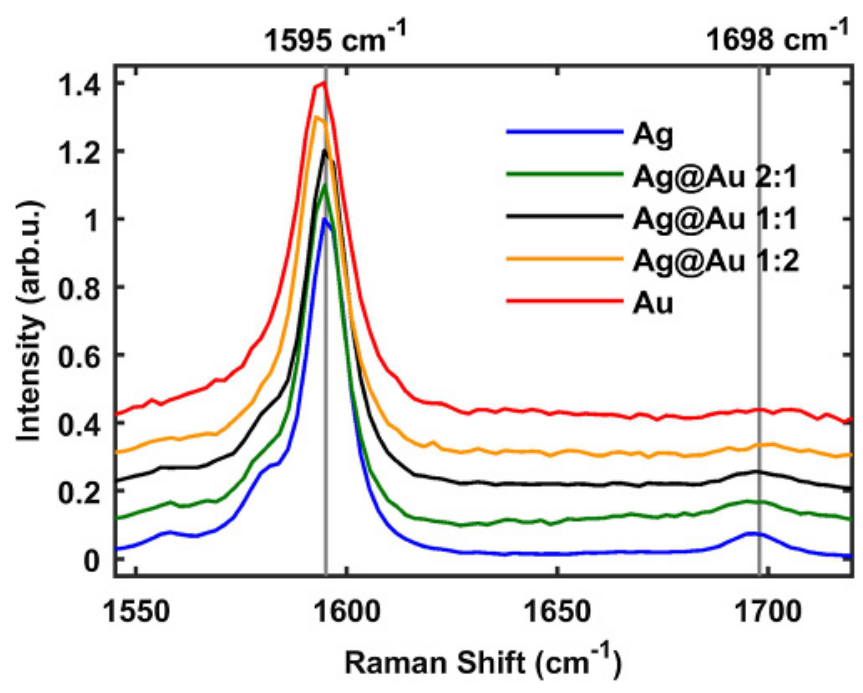

Fig. 6. Zoom into the (normalized) Raman spectrum obtained with the $532 \mathrm{~nm}$ laser in the region of the $1595 \mathrm{~cm}^{-1}$ and $1698 \mathrm{~cm}^{-1}$ modes, which are sensitive to the substrate. It can be seen that the spectra gradually change their character from the case of molecules bound to plain $\mathrm{Ag}$ towards plain $\mathrm{Au}$ nanoparticles with increasing $\mathrm{Au}$ shell thickness.

of small plasmonic nanostructures. It is shown that for deposited Ag@Au core@shell nanoparticles, with diameters of about $5 \mathrm{~nm}$, the spectral position of the localized surface plasmon resonance can be controlled by the $\mathrm{Ag}: \mathrm{Au}$ ratio, which has been adjusted to 2:1, 1:1 and 1:2. Furthermore, upon functionalization with 4-MBT molecules, the nanostructures prepared from these nanoparticles are shown to be suitable substrates for surface-enhanced Raman spectroscopy (SERS). Raman enhancement factors determined for the $1080 \mathrm{~cm}^{-1}$ mode are on the order of $10^{3}-10^{4}$. The Raman spectroscopy results further reveal that only particles with highest Au concentration (Ag: $\mathrm{Au}$ of 1:2) exhibit the signature of a plain Au shell.

In the present work we demonstrate the helium droplet synthesis approach for the production of plasmonic nanoparticles and nanosubstrates with the standard plasmonic materials, Au and Ag as well as bimetallic Ag@Au particles. However, in general the pickup technique allows choosing from a large variety of possible dopant materials. Moreover, the production method allows to produce small core@shell clusters without any chemistry involved, while the $5 \mathrm{~nm}$ size does not even represent a lower limit. The approach can be easily employed to explore novel material combinations for applications in plasmonics [52]. A particularly interesting field for which the approach seems well suited is the production of SERS active substrates composed of nanoparticles with plasmon resonances in the UV, such as aluminum [53], due to the inert synthesis environment and the possibility to protect core materials from oxidation by suitable shell materials [9]. As the helium synthesis approach has recently been employed for the production of nanoparticles interesting for catalysis $[9,54]$, the approach may also enable the production of systems that combine plasmonic with catalytic properties [55].
Open access funding provided by Austrian Science Fund (FWF). The authors gratefully acknowledge support from the Austrian Science Fund (FWF) under grant P30940-N36 as well as support from NAWI Graz. We thank Thomas Jauk for contributions to the UV/vis spectrophotometer measurements and the Institute of Solid State Physics at Graz University of Technology for providing access to the UV/vis spectrophotometer.

Open Access This is an open access article distributed under the terms of the Creative Commons Attribution License (http://creativecommons.org/licenses/by/4.0/), which permits unrestricted use, distribution, and reproduction in any medium, provided the original work is properly cited.

\section{Author contribution statement}

All the authors were involved in the preparation of the manuscript. All the authors have read and approved the final manuscript.

\section{References}

1. M.B. Gawande, A. Goswami, T. Asefa, H. Guo, A.V. Biradar, D.-L. Peng, R. Zboril, R.S. Varma, Chem. Soc. Rev. 44, 7540 (2015)

2. H.A. Atwater, A. Polman, Nat. Mater. 9, 205 (2010)

3. B. Sharma, R.R. Frontiera, A.-I. Henry, E. Ringe, R.P. Van Duyne, Mater. Today 15, 16 (2012)

4. K. Kneipp, M. Moskovits, H. Kneipp, Phys. Today 60, 40 (2007)

5. P.K. Jain, I.H. El-Sayed, M.A. El-Sayed, Nano Today 2, $18(2007)$

6. M.B. Cortie, A.M. McDonagh, Chem. Rev. 111, 3713 (2011)

7. J.P. Toennies, A.F. Vilesov, Angew. Chem. Int. Ed. 43, 2622 (2004)

8. C. Callegari, W.E. Ernst, in Handbook of High-Resolution Spectroscopy, Chapter: Helium Droplets as Nanocryostats for Molecular Spectroscopy-from the Vacuum Ultraviolet to the Microwave Regime, edited by M. Quack, F. Merkt (John Wiley \& Sons, Hoboken, NJ, 2011), pp. 1551-1594

9. M. Schnedlitz, M. Lasserus, R. Meyer, D. Knez, F. Hofer, W.E. Ernst, A.W. Hauser, Chem. Mater. 30, 1113 (2018)

10. M.G. Blaber, M.D. Arnold, M.J. Ford, J. Phys.: Condens. Matter 22, 143201 (2010)

11. Y. Yang, J. Shi, G. Kawamura, M. Nogami, Scr. Mater. 58, 862 (2008)

12. K. Mallik, M. Mandal, N. Pradhan, T. Pal, Nano Lett. 1, 319 (2001)

13. J.H. Hodak, A. Henglein, M. Giersig, G.V. Hartland, J. Phys. Chem. B 104, 11708 (2000)

14. A.K. Samal, L. Polavarapu, S. Rodal-Cedeira, L.M. Liz-Marzán, J. Pérez-Juste, I. Pastoriza-Santos, Langmuir 29, 15076 (2013)

15. S.K. Cha, J.H. Mun, T. Chang, S.Y. Kim, J.Y. Kim, H.M. Jin, J.Y. Lee, J. Shin, K.H. Kim, S.O. Kim, ACS Nano 9, 5536 (2015)

16. Y. Chen, H. Wu, Z. Li, P. Wang, L. Yang, Y. Fang, Plasmonics 7, 509 (2012) 
17. M.S. Shore, J. Wang, A.C. Johnston-Peck, A.L. Oldenburg, J.B. Tracy, Small 7, 230 (2011)

18. M.P. Mallin, C.J. Murphy, Nano Lett. 2, 1235 (2002)

19. B. Baruah, M. Kiambuthi, RSC Adv. 4, 64860 (2014)

20. G. Haberfehlner, P. Thaler, D. Knez, A. Volk, F. Hofer, W.E. Ernst, G. Kothleitner, Nat. Commun. 6, 8779 (2015)

21. M. Lasserus, M. Schnedlitz, D. Knez, R. Messner, A. Schiffmann, F. Lackner, A.W. Hauser, F. Hofer, W.E. Ernst, Nanoscale 10, 2017 (2018)

22. P. Thaler, A. Volk, M. Ratschek, M. Koch, W.E. Ernst, J. Chem. Phys. 140, 044326 (2014)

23. R. Fernández-Perea, L.F. Gómez, C. Cabrillo, M. Pi, A.O. Mitrushchenkov, A.F. Vilesov, M.P. de Lara-Castells, J. Phys. Chem. C 121, 22248 (2017)

24. E. Loginov, L.F. Gomez, A.F. Vilesov, J. Phys. Chem. A 115, 7199 (2011)

25. A. Volk, P. Thaler, M. Koch, E. Fisslthaler, W. Grogger, W.E. Ernst, J. Chem. Phys. 138, 214312 (2013)

26. A. Boatwright, C. Feng, D. Spence, E. Latimer, C. Binns, A.M. Ellis, S. Yang, Faraday Discuss. 162, 113 (2013)

27. S.B. Emery, K.B. Rider, B.K. Little, C.M. Lindsay, J. Phys. Chem. C 117, 2358 (2013)

28. E. Latimer, D. Spence, C. Feng, A. Boatwright, A.M. Ellis, S. Yang, Nano Lett. 14, 2902 (2014)

29. P. Thaler, A. Volk, D. Knez, F. Lackner, G. Haberfehlner, J. Steurer, M. Schnedlitz, W.E. Ernst, J. Chem. Phys. 143, 134201 (2015)

30. L.F. Gomez, E. Loginov, R. Sliter, A.F. Vilesov, J. Chem. Phys. 135, 154201 (2011)

31. P. Thaler, A. Volk, F. Lackner, J. Steurer, D. Knez, W. Grogger, F. Hofer, W.E. Ernst, Phys. Rev. B 90, $155442(2014)$

32. C. Kittel, Introduction to Solid State Physics (Wiley, Hoboken, NJ, 2005)

33. E.C. Le Ru, E. Blackie, M. Meyer, P.G. Etchegoin, J. Phys. Chem. C 111, 13794 (2007)

34. P.H.C. Camargo, L. Au, M. Rycenga, W. Li, Y. Xia, Chem. Phys. Lett. 484, 304 (2010)

35. T. Walther, C.J. Humphreys, J. Cryst. Growth 197, 113 (1999)

36. H. Hövel, S. Fritz, A. Hilger, U. Kreibig, M. Vollmer, Phys. Rev. B 48, 18178 (1993)
37. A. Kuzma, M. Weis, M. Daricek, J. Uhrik, F. Horinek, M. Donoval, F. Uherek, D. Donoval, J. Appl. Phys. 115, 053517 (2014)

38. M. Moskovits, I. Srnová-Šloufová, B. Vlčková, J. Chem. Phys. 116, 10435 (2002)

39. W.-T. Wu, C.-H. Chen, C.-Y. Chiang, L.K. Chau, Sensors 18, 1759 (2018)

40. K.P. Jain, M.A. El-Sayed, Chem. Phys. Lett. 487, 153 (2010)

41. J.A. Scholl, A.L. Koh, J.A. Dionne, Nature 483, 421 (2012)

42. A. Schiffmann, D. Knez, F. Lackner, M. Lasserus, R. Messner, M. Schnedlitz, G. Kothleitner, F. Hofer, W.E. Ernst, J. Appl. Phys. 125, 023104 (2019)

43. T.P. Martin, Phys. Rep. 273, 199 (1996)

44. V. Myroshnychenko, J. Rodríguez-Fernández, I. PastorizaSantos, A.M. Funston, C. Novo, P. Mulvaney, L.M. Liz-Marzán, F.J.G. de Abajo, Chem. Soc. Rev. 37, 1792 (2008)

45. M. Pellarin, M. Broyer, J. Lermé, M.-A. Lebeault, J. Ramade, E. Cottancin, Phys. Chem. Chem. Phys. 18, 4121 (2016)

46. X. Song, G. Wang, Y. Ma, S. Jiang, W. Yue, S. Xu, C. Wang, Chem. Phys. Lett. 645, 164 (2016)

47. O. Endo, M. Nakamura, K. Amemiya, J. Electron. Spectrosc. Relat. Phenom. 187, 72 (2013)

48. A. Ulman, Chem. Rev. 96, 1533 (1996)

49. K. Ikeda, S. Suzuki, K. Uosaki, J. Am. Chem. Soc. 135, 17387 (2013)

50. S.K. Ghosh, S. Nath, S. Kundu, E. Kunio, T. Pal, J. Phys. Chem. B 108, 13963 (2004)

51. F.S. Ameer, Y. Zhou, S. Zou, D. Zhang, J. Phys. Chem. C 118, 22234 (2014)

52. P.R. West, S. Ishii, G.V. Naik, N.K. Emani, V.M. Shalaev, A. Boltasseva, Laser Photonics Rev. 4, 795 (2010)

53. G. Maidecchi, G. Gonella, R. Proietti Zaccaria, R. Moroni, L. Anghinolfi, A. Giglia, S. Nannarone, L. Mattera, H.-L. Dai, M. Canepa, F. Bisio, ACS Nano 7, 5834 (2013)

54. Q. Wu, C.J. Ridge, S. Zhao, D. Zakharov, J. Cen, X. Tong, E. Connors, D. Su, E.A. Stach, C.M. Lindsay, A. Orlov, J. Phys. Chem. Lett. 7, 2910 (2016)

55. S. Linic, P. Christopher, D.B. Ingram, Nat. Mater. 10, 911 (2011) 